\title{
Analysis of Positioning Error for Two-Dimensional Location System
}

\author{
Yi Jiang, ${ }^{1,2}$ Qing Hu, ${ }^{1}$ and Dongkai Yang ${ }^{2}$ \\ ${ }^{1}$ Information Science and Technology College, Dalian Maritime University, Dalian 116026, China \\ ${ }^{2}$ School of Electronic and Information Engineering, Beihang University, Beijing 100083, China \\ Correspondence should be addressed to Yi Jiang; j_y@dlmu.edu.cn
}

Received 4 June 2013; Accepted 11 October 2013

Academic Editor: John Gunnar Carlsson

Copyright (c) 2013 Yi Jiang et al. This is an open access article distributed under the Creative Commons Attribution License, which permits unrestricted use, distribution, and reproduction in any medium, provided the original work is properly cited.

\begin{abstract}
This paper investigates the positioning error using different positioning techniques for two-dimensional location system. Based on the measurement models of TOA with/without the time synchronization and TDOA techniques, the performance comparison analysis between TOA with/without the time synchronization and TDOA is presented respectively. The performance of TOA with the time synchronization and TDOA is influenced by the specific distribution of the reference nodes. Hence, it is hard to determine which one is better. The performance of TDOA is always superior to TOA without the time synchronization using the same reference nodes. These conclusions are finally illustrated by the simulation results presented in two scenarios.
\end{abstract}

\section{Introduction}

Nowadays, numerous systems are available for outdoor localization, such as Global Positioning System (GPS), Instrument Landing System (ILS), and Very High Frequency (VHF) Omnidirectional Range (VOR) [1]. Two-dimensional positioning can offer latitude and longitude information. Threedimensional positioning adds altitude to the two above. In most cases, two-dimensional positioning is sufficient to solve a given task as altitude information is not interesting. Positioning techniques are different by measurement parameters [2], such as the Received Signal Strength (RSS) [3], Angle of Arrival (AOA) [4], and signal propagation time [5-7]. The RSS technique uses the received signal power for positioning. Its accuracy is limited by the fading of wireless signals [3]. The AOA technique requires either directional antennas or antenna arrays to measure the AOA of the signal sent by the mobile to be positioned. The signal propagation time technique estimates the mobile location by measuring the time it takes for the signal to travel from the reference nodes to the mobile. They could achieve very accurate estimation of the mobile location if combined with high-precision timing measurement techniques [8]. The signal propagation time technique has better positioning accuracy than the RSS and
AOA techniques in general [9]. Therefore, this paper focuses mainly on the signal propagation time technique.

The technique based on signal propagation time can be further categorized as follows: Time of Arrival (TOA) $[10,11]$ and Time Difference of Arrival (TDOA) [12]. The TOA technique employs the information of the absolute signal travel time from the reference nodes to the mobile. By multiplying the speed of light, the transmit time between the reference nodes and the mobile can be used to obtain the distances. Based on the geometrical principle, the mobile lies on a circle centered at the reference node's location. The mobile location is determined as the intersection of the circles. The TOA technique can be used in two different cases. The first one requires strict synchronization between the reference nodes and the mobile. It is used in many wireless ad hoc sensor network applications [13]. However, some time the time synchronization is a difficult task to achieve in reality. The other eliminates the need for synchronization between the reference nodes and the mobile and solves the clock bias as an unknown parameter. A well-known application example is Global Navigation Satellite System (GNSS). TDOA is the hyperbolic location technique, which is one of the most widely utilized positioning techniques in cellular mobile communication systems and ship navigation 
systems. In the case of TDOA, only the reference nodes are synchronized, while the mobile does not need to be synchronized. The mobile location is estimated by means of measuring the difference of transmission time from it to a pair of reference nodes. It does not require knowledge of the time that the signal left the transmitter at. For each pair of reference nodes, TDOA yields a hyperbolic curve with the reference node at the foci. The position of the mobile is determined as the intersection of the hyperbolic curves. Loran $\mathrm{C}$ is a typical application. However, its accuracy is not high enough to meet the growing demand [14].

Some studies have focused on the statistical performance analysis of these location techniques. In general, an analysis of this kind makes a number of assumptions about the nature of the available TOA and TDOA parameters $[12,15,16]$. References $[17,18]$ presented dilution of precision (DOP) relationships between TOA for point positioning and TDOA for relative positioning in GPS. References [19-21] showed that the positioning error estimate of TDOA is exactly the same as that of TOA using linearized weighted least square algorithm resulting in the same DOPs. A comparison analysis established in two different ways (strict and average) of the accuracy of various location techniques with the linearized weighted least square estimator has been performed [22]. Most of these existing performance analyses of TOA and TDOA make some assumptions, for example, based on linearized weighted least square algorithm, out of time synchronization, in GPS positioning. As the number of the reference nodes increases, both TOA and TDOA techniques can be used in other positioning applications, such as ground-based navigation. It might be interesting to know which technique makes better accurate positioning with the general algorithm to estimate the mobile location. To tackle this problem, the aim of this paper is to investigate the positioning error of TOA and TDOA with and without the time synchronization using the general linearized least square algorithm. And the performance of these different positioning techniques is also compared.

This paper is organized as follows. In Section 2, the measurement models of TOA and TDOA techniques are explained. The theoretical analysis of the performance comparison between TOA with or without the time synchronization and TDOA is presented in Section 3, respectively. Section 4 consists of the simulation of two scenarios and the results verify the analytic comparison results. Finally, some concluding remarks are given in Section 5.

\section{Measurement Model and Location Estimation}

In the two-dimensional location system, mobile location estimation can be determined using the latitude and longitude coordinates of the reference nodes using TOA and TDOA techniques [23]. $(\varphi, \lambda)$ denote the latitude and longitude coordinates of the mobile. For convenience, the changes in latitude and longitude are denoted by the horizontal and vertical increments $(\Delta \varphi, \Delta \omega)$, as shown in Figure 1.

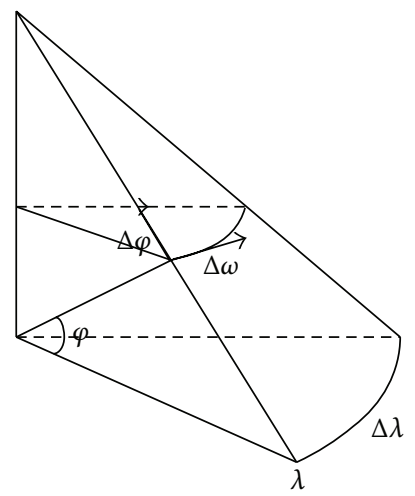

Figure 1: Relationship between $(\varphi, \lambda)$ and $(\Delta \varphi, \Delta \omega)$.

$(\varphi, \lambda)$ and $(\Delta \varphi, \Delta \omega)$ satisfy the following equations:

$$
\begin{gathered}
\varphi^{\prime}=\varphi+\Delta \varphi, \\
\lambda^{\prime}=\lambda+\Delta \omega \sec \varphi .
\end{gathered}
$$

The measurement parameter is indicated by $L$ in the location system. The location equation can be modeled as $L=L(\varphi, \omega)$ that is nonlinear generally. Obtain the linear location equation using Taylor-series, keeping only terms below second order

$$
\bar{L}_{i}=\widehat{L}_{i}+\frac{\partial \widehat{L}_{i}}{\partial \varphi} d \varphi+\frac{\partial \widehat{L}_{i}}{\partial \omega} d \omega,
$$

where $\bar{L}_{i}$ and $\widehat{L}_{i}$ denote the measurement value and its estimation for the $i$ th reference node (or a pair of reference nodes), respectively. Usually the measurements from different reference nodes are obtained at the same time. Stacking all the measurements, (2) can be written in matrix form as follows:

$$
\left[\begin{array}{c}
\delta L_{1} \\
\vdots \\
\delta L_{n}
\end{array}\right]=\left[\begin{array}{cc}
\frac{\partial \widehat{L}_{1}}{\partial \varphi} & \frac{\partial \widehat{L}_{1}}{\partial \omega} \\
\vdots & \vdots \\
\frac{\partial \widehat{L}_{n}}{\partial \varphi} & \frac{\partial \widehat{L}_{n}}{\partial \omega}
\end{array}\right]\left[\begin{array}{c}
d \varphi \\
d \omega
\end{array}\right]
$$

where $\delta L_{i}=\bar{L}_{i}-\widehat{L}_{i}$. As

$$
\begin{aligned}
& \boldsymbol{\alpha}=\left[\begin{array}{lll}
\frac{\partial \widehat{L}_{1}}{\partial \varphi} & \cdots & \frac{\partial \widehat{L}_{n}}{\partial \varphi}
\end{array}\right]^{T}, \\
& \boldsymbol{\beta}=\left[\begin{array}{lll}
\frac{\partial \widehat{L}_{1}}{\partial \omega} & \cdots & \frac{\partial \widehat{L}_{n}}{\partial \omega}
\end{array}\right]^{T}, \\
& \mathbf{d X}=\left[\begin{array}{ll}
d \varphi & d \omega
\end{array}\right]^{T}, \\
& \mathbf{L}=\left[\begin{array}{lll}
\delta L_{1} & \cdots & \delta L_{n}
\end{array}\right]^{T} \text {, }
\end{aligned}
$$

then

$$
L=\left[\begin{array}{ll}
\alpha & \beta
\end{array}\right] \mathbf{d X}
$$


We can write (5) more compactly as

$$
\operatorname{AdX}=\mathbf{L} \text {, }
$$

where

$$
A=\left[\begin{array}{ll}
\alpha & \beta
\end{array}\right]
$$

The least square solution for the corrections to the estimation $(\Delta \varphi, \Delta \omega)$ can be written as

$$
\mathbf{d X}=\left(\mathbf{A}^{T} \mathbf{A}\right)^{-1} \mathbf{A}^{T} \mathbf{L} .
$$

Consequently the latitude and longitude $(\varphi, \lambda)$ of the mobile can be gotten according to (1). Assuming the measurement error is very small, the effects on the linearization of the location equation can be neglected. If the measurement error term in the equation is retained, the location matrix (6) can be rewritten as

$$
\mathbf{A}\left[\mathbf{d X}+\boldsymbol{\varepsilon}_{\mathbf{X}}\right]=\mathbf{L}+\boldsymbol{\varepsilon},
$$

where $\boldsymbol{\varepsilon}$ and $\boldsymbol{\varepsilon}_{\mathbf{x}}$ denote the measurement and positioning error vector, respectively. According to the least square algorithm, the solution of the positioning error vector is given by

$$
\boldsymbol{\varepsilon}_{\mathbf{x}}=\left(\mathbf{A}^{T} \mathbf{A}\right)^{-1} \mathbf{A}^{T} \boldsymbol{\varepsilon}
$$

The above expression shows the relationship between the measurement error and the positioning error. Therefore, in order to determine the mean and variance of the positioning error, a measurement error model needs to be given firstly. In practice, it is not feasible to know the accurate probability distribution of the measurement error. In order to simplify the analysis of the positioning error, we make the following assumptions for the measurement error model.

(a) For all the reference nodes or pairs of reference nodes, the measurement error $\varepsilon$ is a normal distribution with the same mean and variance

$$
\begin{gathered}
E(\varepsilon)=\mathbf{0}, \\
\operatorname{cov}(\varepsilon)=\sigma^{2} \mathbf{I},
\end{gathered}
$$

where $E(\cdot)$ denotes the mean value, $\operatorname{cov}(\cdot)$ denotes the covariance, $\mathbf{I}$ is an identity matrix, and $\sigma$ is the common standard deviation of the measurement error.

(b) Measurement errors between the different reference nodes or pairs of reference nodes are uncorrelated. Thus, the covariance matrix $\mathbf{Q}$ of the measurement error vector is a diagonal matrix

$$
\mathbf{Q}=E\left[(\boldsymbol{\varepsilon}-E(\boldsymbol{\varepsilon}))(\boldsymbol{\varepsilon}-E(\boldsymbol{\varepsilon}))^{T}\right]=\sigma^{2} \mathbf{I}
$$

On the basis of the above two assumptions, the covariance matrix of the positioning error is

$$
\operatorname{cov}\left(\boldsymbol{\varepsilon}_{\mathbf{x}}\right)=E\left(\boldsymbol{\varepsilon}_{\mathbf{x}} \boldsymbol{\varepsilon}_{\mathbf{x}}^{T}\right)=\left(\mathbf{A}^{T} \mathbf{A}\right)^{-1} \sigma^{2}
$$

where $\left(\mathbf{A}^{T} \mathbf{A}\right)^{-1}$ is symmetric, called a weight coefficient matrix. The element contains the geometric accuracy and correlated information of all the solutions. It is the basis of the location accuracy evaluation.
2.1. Measurement Model for TOA. For the TOA technique, according to (4) it can be obtained that

$$
\begin{gathered}
\alpha_{i}=\frac{\partial \widehat{L}_{i}}{\partial \varphi}=-\cos \gamma_{i} \\
\beta_{i}=\frac{\partial \widehat{L}_{i}}{\partial w}=-\sin \gamma_{i} \\
\delta L_{i}=\bar{D}_{i}-\widehat{D}_{i},
\end{gathered}
$$

where $\gamma_{i}$ is the estimated azimuth angle directed from the estimation of the mobile's location to the $i$ th reference node. $\bar{D}_{i}$ and $\widehat{D}_{i}$ represent the measured and estimated distance between the mobile and the $i$ th reference node, respectively. $\gamma_{i}$ and $\widehat{D}_{i}$ can be calculated by the following formulas:

$$
\begin{gathered}
\gamma_{i}=\tan ^{-1}\left(\frac{\cos \varphi_{2} \sin \left(\lambda_{1}-\lambda_{2}\right)}{\cos \varphi_{1} \sin \varphi_{2}-\sin \varphi_{1} \cos \varphi_{2} \cos \left(\lambda_{1}-\lambda_{2}\right)}\right), \\
\widehat{D}_{i}=\cos ^{-1}\left(\sin \varphi_{1} \sin \varphi_{2}+\cos \varphi_{1} \cos \varphi_{2} \cos \left(\lambda_{1}-\lambda_{2}\right)\right) .
\end{gathered}
$$

It should be noted that the unit of the estimated azimuth angle is radians.

If the mobile clock is perfectly synchronized with the clock in the reference node, the time delay from the transmitter to the mobile can be measured precisely. The distance between them will be obtained by multiplying the signal propagation speed. The coordinate of the mobile can be solved having at least two reference nodes. Let

$$
\mathbf{h}=\mathbf{A}=\left[\begin{array}{cc}
-\cos \gamma_{1} & -\sin \gamma_{1} \\
\vdots & \vdots \\
-\cos \gamma_{n} & -\sin \gamma_{n}
\end{array}\right]
$$

Then the positioning error is

$$
\boldsymbol{\varepsilon}_{\mathbf{x T O A}}=\left(\mathbf{h}^{T} \mathbf{h}\right)^{-1} \mathbf{h}^{T} \boldsymbol{\varepsilon} .
$$

The covariance matrix of the positioning error is given by

$$
\operatorname{cov}\left(\boldsymbol{\varepsilon}_{\mathbf{x T O A}}\right)=\left(\mathbf{h}^{T} \mathbf{h}\right)^{-1} \sigma^{2}
$$

The time synchronization, however, is a difficult task to achieve in reality. In the TOA technique, the clock bias augments the two-dimensional location vector forming a three-dimensional state vector. Thus, to estimate the mobile location, more than three independent TOA measurements are required. Equation (2) is rewritten as

$$
\bar{L}_{i}=\widehat{L}_{i}+\frac{\partial \widehat{L}_{i}}{\partial x} d \varphi+\frac{\partial \widehat{L}_{i}}{\partial y} d \omega+d T
$$


where $d T=c \cdot d t . d t$ is the mobile clock bias. $c$ is the signal propagation speed, equaling the speed of light. Write the location equations in matrix form

$$
\left[\begin{array}{c}
\delta L_{1} \\
\vdots \\
\delta L_{n}
\end{array}\right]=\left[\begin{array}{ccc}
\frac{\partial \widehat{L}_{1}}{\partial \varphi} & \frac{\partial \widehat{L}_{1}}{\partial \omega} & 1 \\
\vdots & \vdots & \vdots \\
\frac{\partial \widehat{L}_{n}}{\partial \varphi} & \frac{\partial \widehat{L}_{n}}{\partial \omega} & 1
\end{array}\right]\left[\begin{array}{c}
d \varphi \\
d \omega \\
d T
\end{array}\right] .
$$

According to (4), the above matrix can be simplified as

$$
\mathbf{L}=\left[\begin{array}{lll}
\boldsymbol{\alpha} & \boldsymbol{\beta} & \mathbf{e}_{n}
\end{array}\right]\left[\begin{array}{l}
\mathbf{d X} \\
d T
\end{array}\right]=\mathbf{H d X}^{\prime},
$$

where

$$
\begin{gathered}
\mathbf{e}_{n}=\left[\begin{array}{lll}
1 & \cdots & 1
\end{array}\right]^{T}, \\
\mathbf{H}=\left[\begin{array}{ll}
\mathbf{A} & \mathbf{e}_{n}
\end{array}\right], \\
\mathbf{d X}^{\prime}=\left[\begin{array}{ll}
\mathbf{d X} & d T
\end{array}\right]^{T} .
\end{gathered}
$$

Using the least square algorithm, the solutions including a clock bias are

$$
\mathbf{d} \mathbf{X}^{\prime}=\left(\mathbf{H}^{T} \mathbf{H}\right)^{-1} \mathbf{H}^{T} \mathbf{L} .
$$

Similar to the TOA technique with the time synchronization, if the measurement errors in the location equations are reserved, the positioning matrix can be rewritten as

$$
\mathbf{H}\left[\mathbf{d X}^{\prime}+\boldsymbol{\varepsilon}_{\mathrm{xTOA}}^{\prime}\right]=\mathbf{L}+\boldsymbol{\varepsilon}
$$

where

$$
\boldsymbol{\varepsilon}_{\mathrm{xtTOA}}^{\prime}=\left[\begin{array}{ll}
\boldsymbol{\varepsilon}_{\mathbf{x T O A}} & \varepsilon_{t}
\end{array}\right]^{T} .
$$

The solution using the least square algorithm is

$$
\boldsymbol{\varepsilon}_{\mathrm{xt} \mathrm{TOA}}^{\prime}=\left(\mathbf{H}^{T} \mathbf{H}\right)^{-1} \mathbf{H}^{T} \boldsymbol{\varepsilon} .
$$

The covariance matrix of the positioning error is

$$
\operatorname{cov}\left(\boldsymbol{\varepsilon}_{\mathbf{x} t \mathrm{TOA}}^{\prime}\right)=\left(\mathbf{H}^{T} \mathbf{H}\right)^{-1} \sigma^{2} .
$$

2.2. Measurement Model for TDOA. As compared with the TOA technique that directly estimates the clock bias included in the state vector, the TDOA technique eliminates the clock bias from the state vector. For the purpose, one element of a TOA measurement vector is selected as the main reference node and the remaining elements are subtracted by this main reference node, resulting in a TDOA measurement vector. According to (4), we can know that

$$
\begin{gathered}
\alpha_{i}=\frac{\partial \widehat{L}_{i}}{\partial \varphi}=-\cos \gamma_{m}+\cos \gamma_{i}, \\
\beta_{i}=\frac{\partial \widehat{L}_{i}}{\partial w}=-\sin \gamma_{m}+\sin \gamma_{i} \\
\delta L_{i}=\Delta \bar{D}_{m i}-\Delta \widehat{D}_{m i},
\end{gathered}
$$

where

$$
\begin{aligned}
\Delta \bar{D}_{m i} & =\bar{D}_{m}-\bar{D}_{i}, \\
\Delta \widehat{D}_{m i} & =\widehat{D}_{m}-\widehat{D}_{i} .
\end{aligned}
$$

The subscript $m$ represents the main reference node. In this paper, the first reference node is selected as the main reference node; that is, $m=1$. Then

$$
\mathbf{G}=\mathbf{A}=\left[\begin{array}{cc}
-\cos \gamma_{1}+\cos \gamma_{2} & -\sin \gamma_{1}+\sin \gamma_{2} \\
\vdots & \vdots \\
-\cos \gamma_{1}+\cos \gamma_{n} & -\sin \gamma_{1}+\sin \gamma_{n}
\end{array}\right] .
$$

The positioning error and its covariance matrix are given by

$$
\begin{gathered}
\boldsymbol{\varepsilon}_{\mathbf{x} T D O A}=\left(\mathbf{G}^{T} \mathbf{G}\right)^{-1} \mathbf{G}^{T} \varepsilon, \\
\operatorname{Cov}\left(\boldsymbol{\varepsilon}_{\mathbf{x} T D O A}\right)=\left(\mathbf{G}^{T} \mathbf{G}\right)^{-1} \sigma^{2} .
\end{gathered}
$$

\section{Positioning Error Comparison}

Comparison of positioning errors for different methods is to compare their covariance matrixes. According to the previous theoretical analysis, we can conclude the following results:

$$
\begin{aligned}
\operatorname{cov}\left(\boldsymbol{\varepsilon}_{\mathbf{x} T O A}\right) & =\left(\mathbf{h}^{T} \mathbf{h}\right)^{-1} \sigma^{2}, \\
\operatorname{cov}\left(\boldsymbol{\varepsilon}_{\mathbf{x t T O A}}^{\prime}\right) & =\left(\mathbf{H}^{T} \mathbf{H}\right)^{-1} \sigma^{2}, \\
\operatorname{cov}\left(\boldsymbol{\varepsilon}_{\mathbf{x T D O A}}\right) & =\left(\mathbf{G}^{T} \mathbf{G}\right)^{-1} \sigma^{2} .
\end{aligned}
$$

Assuming that the same measurement mechanism is used for both TOA and TDOA techniques, the variances of the measurement errors are equal. Therefore the comparison of the positioning errors is to compare the weight coefficient matrixes. In Automatic Identification System (AIS), the mobile can only receive positioning signals from three reference nodes at the same time, which will be focused on in this paper.

3.1. Comparison of TOA with the Time Synchronization and TDOA. From (16) and (30), we can derive

$$
\mathbf{G}=\mathbf{S h} .
$$

$\mathbf{S}$ is called a transformation matrix defined as follows:

$$
\mathbf{S}=\left[\begin{array}{ll}
\mathbf{e}_{2} & -\mathbf{I}_{2}
\end{array}\right] .
$$

$\mathbf{I}_{2}$ is the identity matrix of order 2 . The covariance matrix of TDOA is rewritten as

$$
\operatorname{cov}\left(\boldsymbol{\varepsilon}_{\mathbf{x T D O A}}\right)=\left(\mathbf{h}^{T} \boldsymbol{S}^{T} \mathbf{S h}\right)^{-1} \sigma^{2}=\left(\mathbf{h}^{T} \mathbf{h}+\mathbf{h}^{T} \mathbf{P h}\right)^{-1} \sigma^{2},
$$

where

$$
\mathbf{P}=\left[\begin{array}{ccc}
1 & -1 & -1 \\
-1 & 0 & 0 \\
-1 & 0 & 0
\end{array}\right]
$$


According to the matrix inversion lemma,

$$
\begin{aligned}
\operatorname{cov}\left(\boldsymbol{\varepsilon}_{\mathbf{x T D O A}}\right) & =\left(\mathbf{h}^{T} \mathbf{h}\right)^{-1} \sigma^{2}+\mathbf{D} \sigma^{2} \\
& =\operatorname{cov}\left(\boldsymbol{\varepsilon}_{\mathbf{x T O A}}\right)+\mathbf{D} \sigma^{2} .
\end{aligned}
$$

The matrix $\mathbf{D}$ could not be decided to be either a positive definite matrix or a negative definite matrix. So it is hard to determine the performance of TOA with the time synchronization being better than or worse than the TDOA technique. It is determined by the specific distribution of the reference nodes.

3.2. Comparison of TOA without the Time Synchronization and TDOA. According to $\mathbf{H}$, as shown in (22), we know that

$$
\mathbf{H}^{T} \mathbf{H}=\left[\begin{array}{cc}
\mathbf{h}^{T} \mathbf{h} & \mathbf{h}^{T} \mathbf{e}_{3} \\
\mathbf{e}_{3}^{T} \mathbf{h} & 3
\end{array}\right] .
$$

According to the inverse of the block matrix,

$$
\left(\mathbf{H}^{T} \mathbf{H}\right)^{-1}=\left[\begin{array}{cc}
3\left(\mathbf{G}^{T} \mathbf{J G}\right)^{-1} & -\left(\mathbf{G}^{T} \mathbf{J G}\right)^{-1} \mathbf{h}^{T} \mathbf{e}_{3} \\
-\mathbf{e}_{3}^{T} \mathbf{h}\left(\mathbf{G}^{T} \mathbf{J G}\right)^{-1} & \frac{1}{3}+\frac{1}{3} \mathbf{e}_{3}^{T} \mathbf{h}\left(\mathbf{G}^{T} \mathbf{J G}\right)^{-1} \mathbf{h}^{T} \mathbf{e}_{3}
\end{array}\right],
$$

where

$$
\mathbf{J}=\left[\begin{array}{cc}
2 & -1 \\
-1 & 2
\end{array}\right]
$$

The positioning error for TOA without the time synchronization is only determined by the matrix $\left(\mathbf{G}^{T} \mathbf{J G}\right)^{-1}$. The covariance matrix of the positioning error is

$$
\operatorname{cov}\left(\boldsymbol{\varepsilon}_{\mathbf{x} T O A}^{\prime}\right)=3\left(\mathbf{G}^{T} \mathbf{J G}\right)^{-1} \sigma^{2}
$$

Hence

$$
\begin{aligned}
\operatorname{cov} & \left(\boldsymbol{\varepsilon}_{\mathbf{x T O A}}^{\prime}\right)-\operatorname{cov}\left(\boldsymbol{\varepsilon}_{\mathbf{x T D O A}}\right) \\
& =\left[3\left(\mathbf{G}^{T} \mathbf{J G}\right)^{-1}-\left(\mathbf{G}^{T} \mathbf{G}\right)^{-1}\right] \sigma^{2} \\
& =\frac{1}{\left|\mathbf{G}^{T} \mathbf{G}\right|}\left[\begin{array}{ll}
c_{11} & c_{12} \\
c_{21} & c_{22}
\end{array}\right] \sigma^{2},
\end{aligned}
$$

where

$$
\begin{aligned}
c_{11} & =\sin ^{2} \gamma_{2}+\sin ^{2} \gamma_{3}-2 \sin \gamma_{2} \sin \gamma_{3} \\
& =\left(\sin \gamma_{2}-\sin \gamma_{3}\right)^{2} \geq 0, \\
c_{22} & =\cos ^{2} \gamma_{2}+\cos ^{2} \gamma_{3}-2 \cos \gamma_{2} \cos \gamma_{3} \\
& =\left(\cos \gamma_{2}-\cos \gamma_{3}\right)^{2} \geq 0 .
\end{aligned}
$$

Thus, the positioning error of TOA without the time synchronization is always greater than TDOA with the same reference nodes. The performance of TDOA is always better than TOA without the time synchronization.

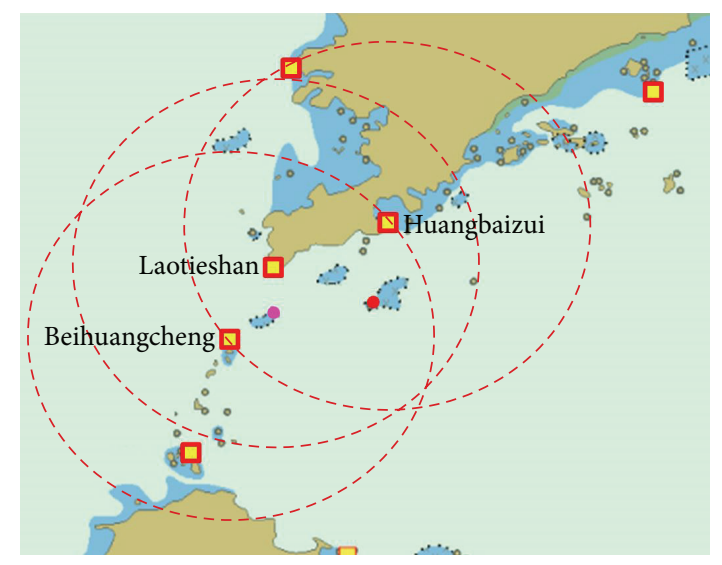

FIgURE 2: Map of AIS reference nodes and mobile locations.

TABLE 1: Information of the AIS reference nodes and mobile locations.

\begin{tabular}{lccc}
\hline & MMSI & Latitude & Longitude \\
\hline Laotieshan & 4131101 & $38^{\circ} 43.6420^{\prime} \mathrm{N}$ & $121^{\circ} 08.1330^{\prime} \mathrm{E}$ \\
Huangbaizui & 4131104 & $38^{\circ} 54.2850^{\prime} \mathrm{N}$ & $121^{\circ} 42.9500^{\prime} \mathrm{E}$ \\
Beihuangcheng & 4131504 & $38^{\circ} 23.6880^{\prime} \mathrm{N}$ & $120^{\circ} 54.6137^{\prime} \mathrm{E}$ \\
Mobile location 1 & & $38^{\circ} 34.4140^{\prime} \mathrm{N}$ & $121^{\circ} 38.1870^{\prime} \mathrm{E}$ \\
Mobile location 2 & & $38^{\circ} 30.0000^{\prime} \mathrm{N}$ & $121^{\circ} 09.0000^{\prime} \mathrm{E}$ \\
\hline
\end{tabular}

\section{Simulations}

To verify the conclusions in Section 3, two types of simulation models are given, which are implemented using Matlab. One is the positioning error analysis of some certain mobile locations using AIS reference nodes named Laotieshan, Huangbaizui, and Beihuangcheng in the real situation. In Figure 2, the yellow box indicates the location of the AIS reference node. Assuming the radius of signal propagation is 45 nautical miles, the signal coverage is shown as a red circle. Two mobile locations are selected for simulation. A red dot indicates the location of the first mobile. A purple dot indicates the other.

Table 1 gives information about three AIS reference nodes, including the latitude and longitude coordinates and their maritime mobile communications service identity (MMSI). The location coordinates of the two selected mobiles are also shown in Table 1.

The covariance matrixes of the positioning error using three different positioning techniques are as follows.

For TOA with the time synchronization

$$
\operatorname{cov}\left(\boldsymbol{\varepsilon}_{\mathbf{x T O A}}\right)=\left[\begin{array}{cc}
0.8461 & -0.0564 \\
-0.0564 & 0.5562
\end{array}\right] \sigma^{2} .
$$

For TOA without the time synchronization

$$
\operatorname{cov}\left(\varepsilon_{\mathrm{xTOA}}^{\prime}\right)=\left[\begin{array}{cc}
4.8358 & -4.1146 \\
-4.1146 & 4.6841
\end{array}\right] \sigma^{2} .
$$

For TDOA

$$
\operatorname{cov}\left(\boldsymbol{\varepsilon}_{\mathrm{xTDOA}}\right)=\left[\begin{array}{cc}
2.3638 & -1.3369 \\
-1.3369 & 1.5630
\end{array}\right] \sigma^{2} .
$$




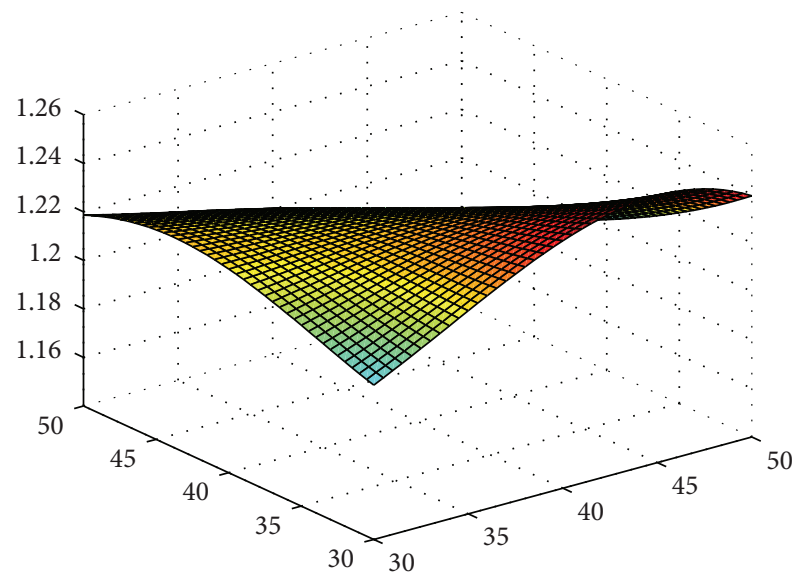

(a)

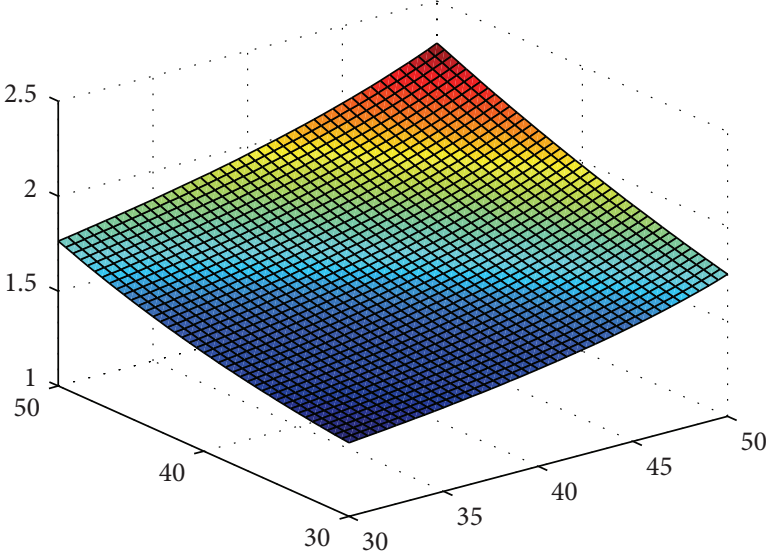

(b)

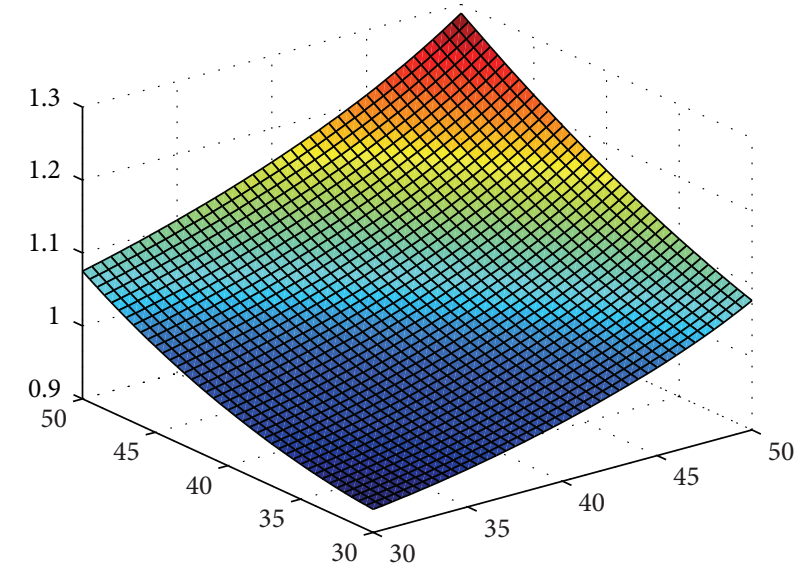

(c)

FIGURE 3: Trace of covariance matrixes with different positioning techniques.

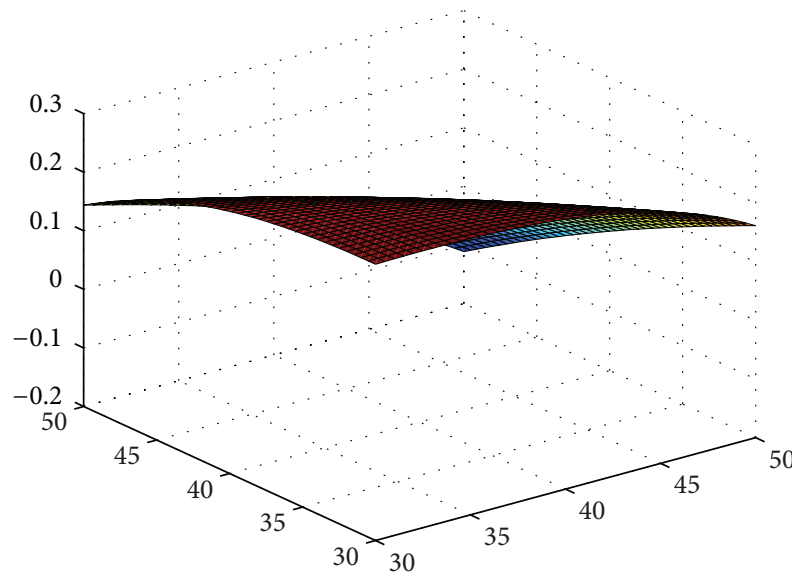

(a)

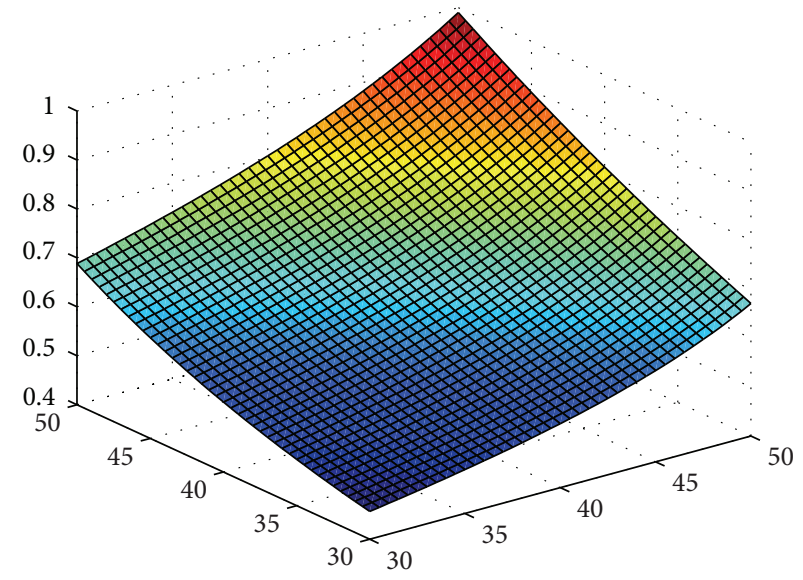

(b)

FIgURE 4: Comparison of the covariance matrixes. 
TABLE 2: Weight coefficient matrix trace using different positioning techniques.

\begin{tabular}{lccc}
\hline Mobile & $\begin{array}{c}\text { TOA with the time } \\
\text { synchronization }\end{array}$ & $\begin{array}{c}\text { TOA without the time } \\
\text { synchronization }\end{array}$ & TDOA \\
\hline Location 1 & 1.184 & 3.085 & 1.982 \\
Location 2 & 1.440 & 1.921 & 1.328 \\
\hline
\end{tabular}

The traces of weight coefficient matrixes of the first mobile location are summarized in Table 2. With the same processing, the covariance matrixes and the corresponding weight coefficient matrix traces of the other mobile location can also be calculated, referring to Table 2 .

Based on these above results, we can draw the following two conclusions. Firstly, in the case of the time synchronization, for the first mobile location, the performance of TOA is superior to TDOA. This is contrasted with the other mobile location, for which the performance of TDOA is better. However, if time is not synchronized, TDOA always has the better performance than TOA. The theoretical derived results are confirmed in Section 3.

It gives the simulation results with some certain mobile locations in the above. Here is the other scenario. Reference nodes are located at $\left(0^{\circ} \mathrm{N}, 0^{\circ} \mathrm{E}\right),\left(60^{\circ} \mathrm{N}, 0^{\circ} \mathrm{E}\right)$, and $\left(0^{\circ} \mathrm{N}, 60^{\circ} \mathrm{E}\right)$. The true mobile locations are assumed at each of $40 \times 40$ grid points whose latitude and longitude are between $30^{\circ}$ and $\sim 50^{\circ}$. The gap between two adjacent grid points is $30^{\prime}$ in either latitude or longitude direction. Figure 3 shows the traces of the covariance matrixes with different positioning techniques when the measurement error variance is 1 . TOA with and without the time synchronization is shown in Figures 3(a) and 3(b), respectively. Figure 3(c) corresponds to the trace of the covariance matrix of TDOA.

The comparison of covariance matrixes using TOA with the time synchronization and TDOA is depicted in Figure 4(a). The difference between TOA and TDOA is greater than or less than 0 sometimes. That is to say, the performance is determined by the distribution of the reference nodes when TOA with the time synchronization and TDOA are compared. Figure 4(b) shows the comparison of the covariance matrixes between TOA without the time synchronization and TDOA. It can be seen from Figure 4(b) that the positioning error of TOA without the time synchronization is always greater than TDOA. Thus simulation results verify the theoretical analysis results.

\section{Conclusions}

The performance of different positioning techniques for twodimensional location system is investigated in this paper. The measurement models of TOA with/without the time synchronization and TDOA techniques are given. Positioning errors of TOA with/without the time synchronization and TDOA are analyzed and compared. With the performance of TOA with the time synchronization and TDOA it is hard to determine which one is better; that is, it depends on the distribution of the reference nodes. The performance of TDOA is always superior to TOA without the time synchronization having the same reference nodes. Finally, these theoretical analysis conclusions are demonstrated by the simulation results.

\section{Acknowledgments}

This work was supported by the Chinese National Science Foundation (No. 61231006), the National Key Technologies R\&D Program of China (No. 2012BAH36B02) and the Fundamental Research Funds for the Central Universities of China (No. 3132013307).

\section{References}

[1] Department of Defense, Federal Radio Navigation Plan, Department of Defense, Virginia Beach, Va, USA, 2010.

[2] M. R. Gholami, S. Gezici, and E. G. Ström, "Improved position estimation using hybrid TW-TOA and TDOA in cooperative networks," IEEE Transactions on Signal Processing, vol. 60, no. 7, pp. 3770-3785, 2012.

[3] A. J. Weiss, "On the accuracy of a cellular location system based on RSS measurements," IEEE Transactions on Vehicular Technology, vol. 52, no. 6, pp. 1508-1518, 2003.

[4] Y. Zhu, D. Huang, and A. Jiang, "Network localization using angle of arrival," in Proceedings of the IEEE International Conference on Electro/Information Technology (IEEE EIT '08), pp. 205-210, May 2008.

[5] S. Gezici, Z. Tian, G. B. Giannakis et al., "Localization via ultrawideband radios: a look at positioning aspects of future sensor networks," IEEE Signal Processing Magazine, vol. 22, no. 4, pp. 70-84, 2005.

[6] I. Güvenç and C. C. Chong, "A survey on TOA based wireless localization and NLOS mitigation techniques," IEEE Communications Surveys and Tutorials, vol. 11, no. 3, pp. 107-124, 2009.

[7] J. Xu, M. Ma, and C. L. Law, "Position estimation using UWB TDOA measurements," in Proceedings of the IEEE International Conference on Ultra-Wideband (ICUWB '06), pp. 605-610, September 2006.

[8] K. Yu, J. Montillet, A. Rabbachin, P. Cheong, and I. Oppermann, "UWB location and tracking for wireless embedded networks," Signal Processing, vol. 86, no. 9, pp. 2153-2171, 2006.

[9] H. L. Jhi, J. C. Chen, C. H. Lin, and C. T. Huang, "A factor-graphbased TOA location estimator," IEEE Transactions on Wireless Communications, vol. 11, no. 5, pp. 1764-1773, 2012.

[10] J. Zhen, X. Ma, and S. Zhang, "TOA-based combined location positioning algorithm in NLOS propagation environment," Journal of Dalian University of Technology, vol. 45, no. 4, pp. 599-602, 2005.

[11] J. Shen, A. F. Molisch, and J. Salmi, "Accurate passive location estimation using TOA measurements," IEEE Transactions on Wireless Communications, vol. 11, no. 6, pp. 2184-2192, 2012.

[12] H. K. Lee, H. S. Kim, J. Y. Shim, and M. B. Heo, "Analytic equivalence of iterated TOA and TDOA techniques under structured measurement characteristics," Multidimensional Systems and Signal Processing, vol. 22, no. 4, pp. 361-377, 2011.

[13] S. Zhu and Z. Ding, "Joint synchronization and localization using TOAs: a linearization based WLS solution," IEEE Journal on Selected Areas in Communications, vol. 28, no. 7, pp. 1016$1025,2010$. 
[14] Z. Han, Q. Wu, D. Song, and D. Gu, "Application of mathematics method on satellite positioning," International Journal of Digital Content Technology and Its Applications, vol. 5, no. 5, pp. 238249, 2011.

[15] A. Abrardo, G. Benelli, C. Maraffon, and A. Toccafondi, "Performance of TDoA-based radiolocation techniques in CDMA urban environments," in Proceedings of the IEEE International Conference on Communications (ICC '02), vol. 1, pp. 431-435, May 2002.

[16] M. Deffenbaugh, J. G. Bellingham, and H. Schmidt, "Relationship between spherical and hyperbolic positioning," in Proceedings of the MTS/IEEE Oceans Conference, vol. 2, pp. 590-595, September 1996.

[17] R. O. Nielsen, "Relationship between dilution of precision for point positioning and for relative positioning with GPS," IEEE Transactions on Aerospace and Electronic Systems, vol. 33, no. 1, pp. 333-338, 1997.

[18] P. J. G. Teunissen, “A proof of Nielsen's conjecture on the GPS dilution of precision," IEEE Transactions on Aerospace and Electronic Systems, vol. 34, no. 2, pp. 693-695, 1998.

[19] C. Park and I. Kim, "Comments on relationships between dilution of precision for point positioning and for relative positioning with GPS," IEEE Transactions on Aerospace and Electronic Systems, vol. 36, no. 1, pp. 315-316, 2000.

[20] D. H. Shin and T. K. Sung, "Comparisons of error characteristics between TOA and TDOA positioning," IEEE Transactions on Aerospace and Electronic Systems, vol. 38, no. 1, pp. 307-311, 2002.

[21] D. H. Shin and T. K. Sung, "Analysis of positioning errors in radionavigation systems," in Proceedings of the IEEE Intelligent Transportation Systems Conference, pp. 156-159, August 2001.

[22] A. Urruela, J. Sala, and J. Riba, "Average performance analysis of circular and hyperbolic geolocation," IEEE Transactions on Vehicular Technology, vol. 55, no. 1, pp. 52-66, 2006.

[23] Y. Jiang, S. Zhang, and D. Yang, "A general method for evaluation of position accuracy," ICIC Express Letters, vol. 7, no. 7, pp. 2035-2040, 2013. 


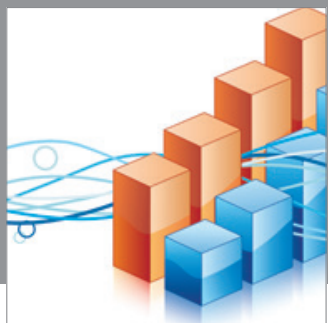

Advances in

Operations Research

mansans

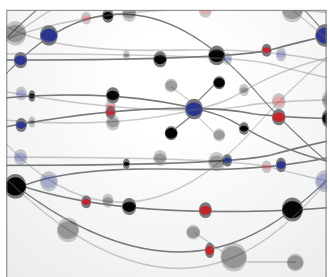

The Scientific World Journal
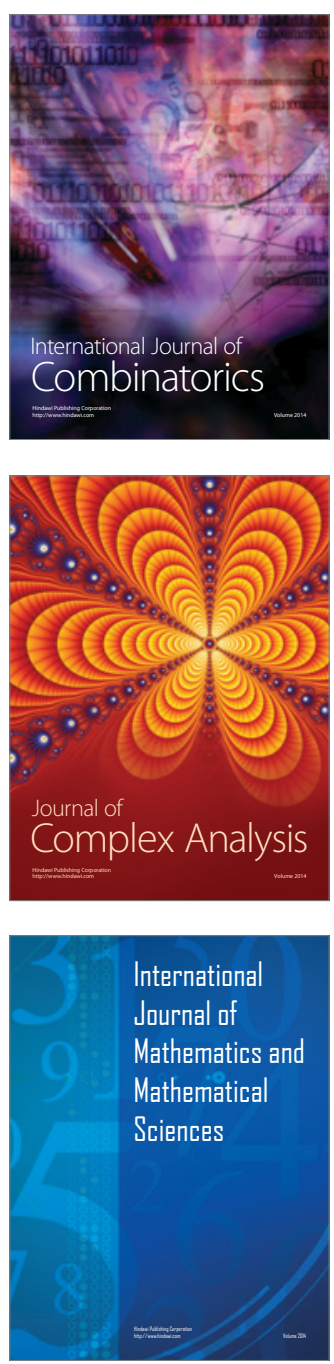
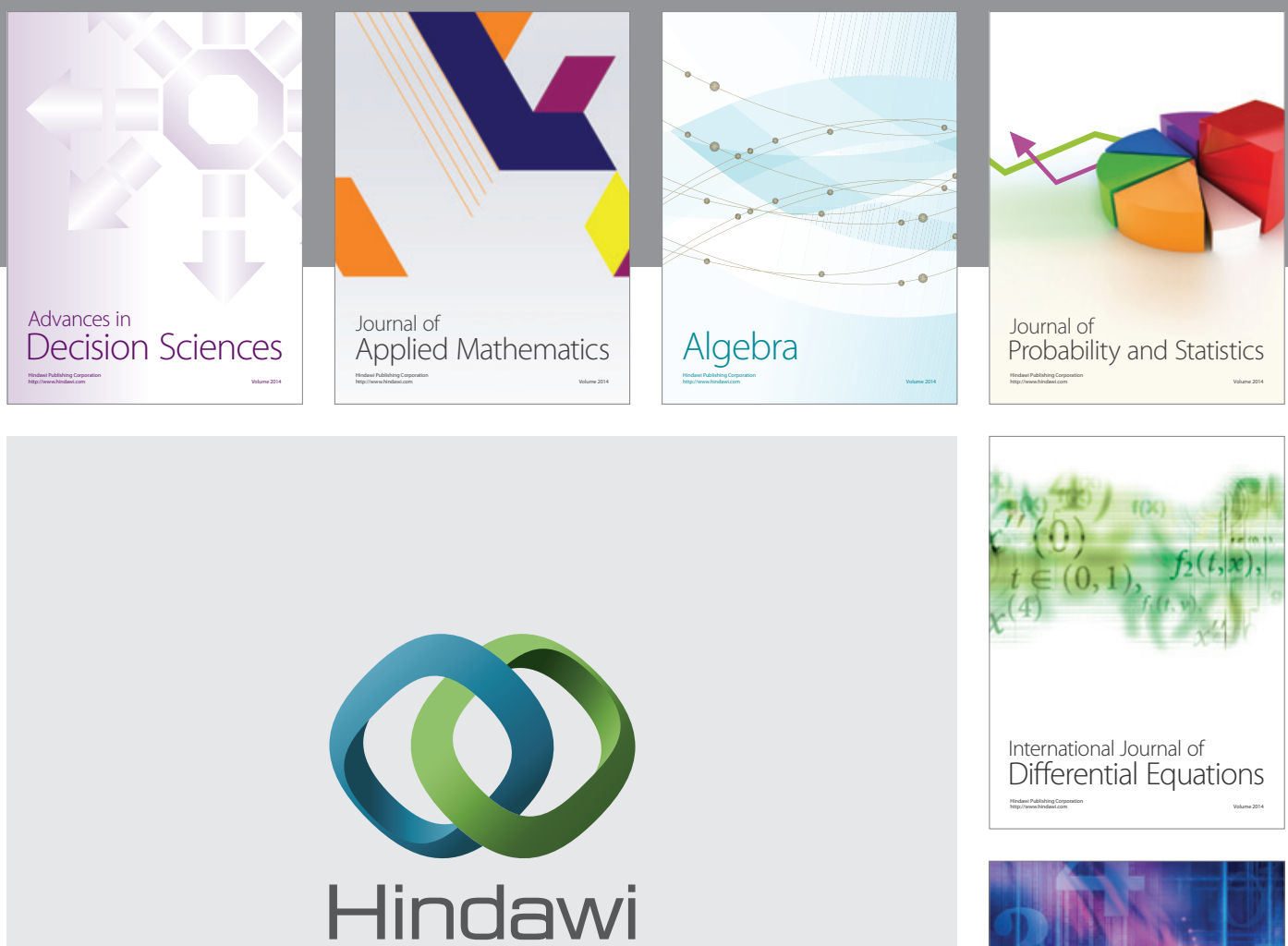

Submit your manuscripts at http://www.hindawi.com
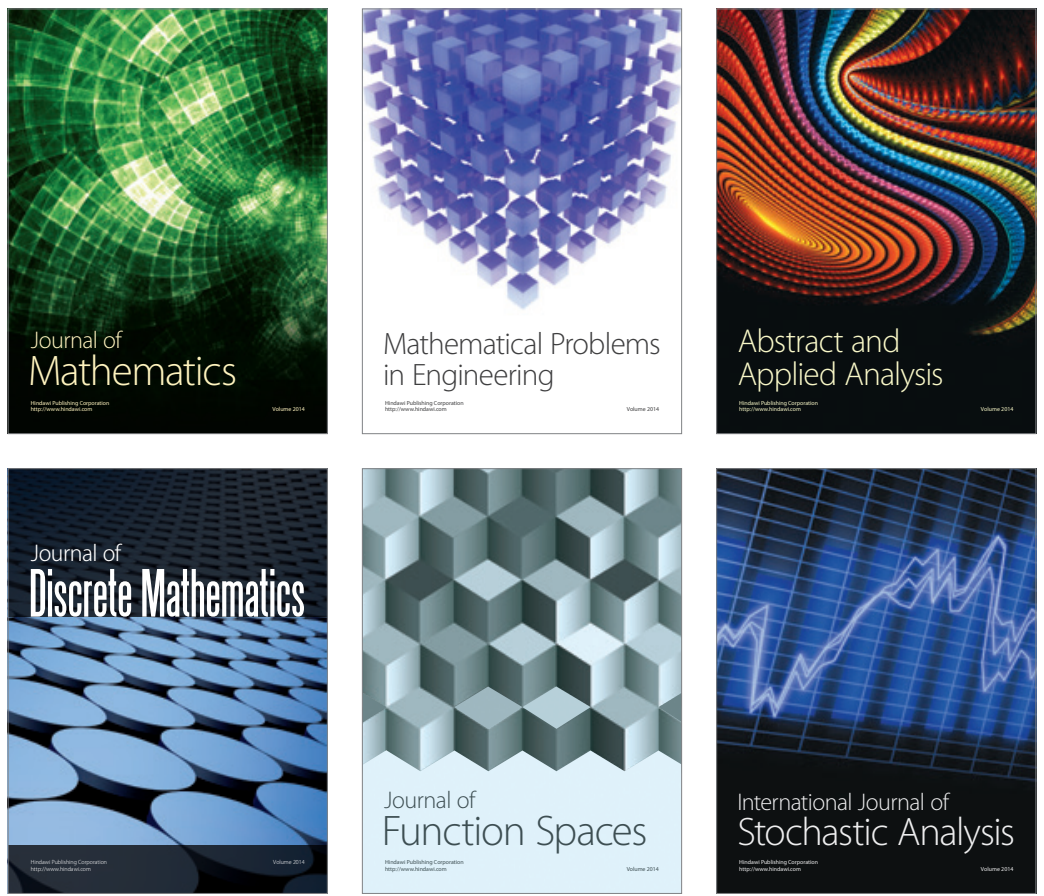

Journal of

Function Spaces

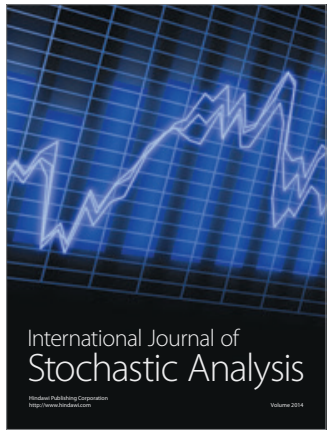

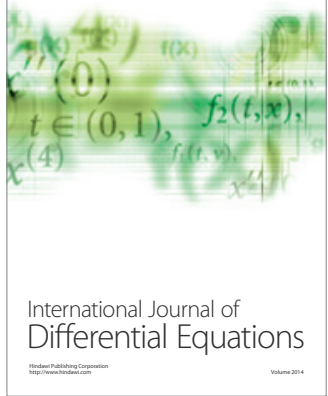
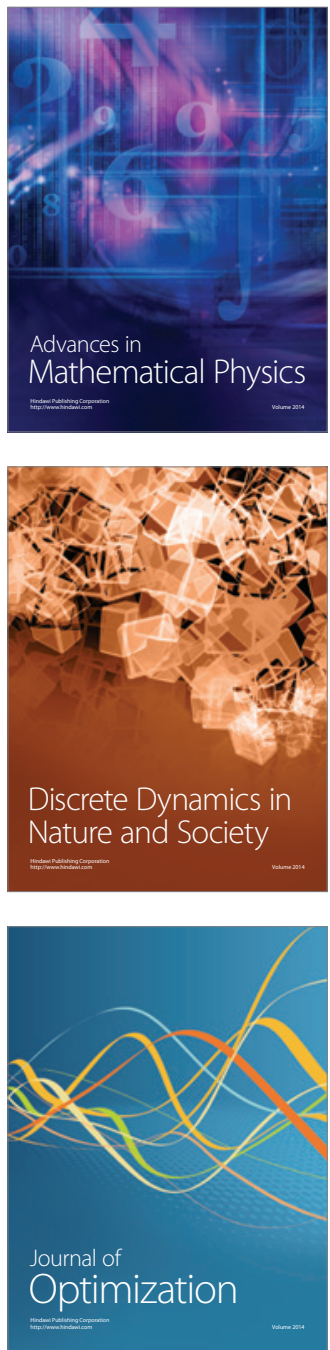